\title{
Microscopy of Chemical and Mechanical Heterogeneities in Lithium Cobalt Oxide
}

\author{
David R. Diercks ${ }^{1}$, Matthew Musselman ${ }^{1}$, Mukesh Kumar ${ }^{1}$, Brian P. Gorman ${ }^{1}$, Corinne E. Packard ${ }^{1,2}$ \\ 1. Colorado School of Mines, Department of Metallurgical and Materials Engineering, Golden, CO USA \\ 2. National Renewable Energy Laboratory, Golden, CO USA
}

Lithium ion batteries (LIB) are used in numerous portable electronic devices primarily due to their light weight and relatively high energy densities. During cycling, they are exposed to both chemical and mechanical gradients. The repetitive action of these can lead to irrecoverable loss of capacity. Both intra- and inter-granular failures arising from Li intercalation induced stresses have been observed [1,2]. These may result from both anisotropies in the strain field as well as anisotropies in the crystal properties [3-5]. Particles that are fractured may become separated from the primary electrical percolation pathway, meaning that this material can no longer participate in the charging and discharging cycles [6,7]. Additionally, solid electrolyte interphases, which bind Li, may be formed at the freshly created surfaces [8]. Lithium cobalt oxide is among the most common LIB cathode materials with a relatively modest volume change during cycling [9]. A transmission electron microscopy (TEM) study of this material has shown a preferred plane for dislocation formation [10] and nanomechanical measurements have indicated orientation-dependent values [11]. However, there have been relatively few studies regarding the nanoscale chemistry and fracture of $\mathrm{LiCoO}_{2}$, particularly for cycled material. A more complete understanding of the nature and interaction of the chemical and mechanical effects could lead to LIB designs and materials that have greater resistance to capacity fade.

In the present work, $\mathrm{LiCoO}_{2}$ particles were taken from a commercial cell that was charged at a $1 \mathrm{C}$ rate to $4.2 \mathrm{~V}$ and discharged at a $3 \mathrm{C}$ rate to $2.75 \mathrm{~V}$ for 1000 cycles resulting in $72 \%$ of its original capacity. These were mounted and polished to expose the cross-sections of the particles. Nanoindentation of the particles followed by scanning electron microscopy analysis revealed fracturing of many of the particles, preventing determination of the mechanical response of the particles. The fractures, while occurring in the regions around the indentations, did not always originate from the regions of highest stress (Figure 1a). Focused ion beam cross-sectioning revealed that the cracks often propagated through entire particles as shown in Figure 1b. TEM diffraction analyses indicated both intra- and inter-granular fracture along $\{001\}$ planes. Inter-granular fracture terminated at grain boundaries. Atom probe tomography (APT) performed on a Cameca LEAP 4000X Si instrument was used to characterization the nanoscale compositions. Uncycled specimens were studied to establish analysis conditions which produced the expected stoichiometry. From these assessments, base temperatures of $35-40 \mathrm{~K}$ and laser energies of $1-2 \mathrm{pJ}$ were used for composition analyses of the cycled material. TEM imaging of the specimens before and after APT analysis (Figure 2a) was used to assist in generating the reconstructions [12]. From the APT analyses, some cycled particles were found to have the stoichiometric Li composition while others were found to be deficient in Li. Analyses across grain boundaries indicated differences in Li concentration on either side of the boundaries. In those cases, one side was found to be enriched in Li, suggesting poor transport into the adjacent grain. The above results indicate that both mechanical and chemical mechanisms played a role in the observed LIB capacity fade [13].

\section{References:}


[1] M. Wohlfahrt-Mehrens, C. Vogler and J. Garche, J. Power Sources, 127 (2004), p. 58.

[2] D. Y. Wang et al., J. Power Sources, 140 (2005), p. 125.

[3] S. Yamakawa et al., J. of Power Sources, 223 (2013), p. 199.

[4] V. Malavé et al., Electrochimica Acta, 130 (2014), p. 707.

[5] D.-W. Chung et al., J. of the Electrochem. Soc., 158 (2011), p. A1083.

[6] X. Zhang, W. Shyy and A. Marie Sastry, J. of the Electrochem. Soc., 154 (2007), p. A910.

[7] Y.-T. Cheng and M. W. Verbrugge, J. of the Electrochem. Soc., 157 (2010), p. A508.

[8] R. Deshpande et al., J. of the Electrochem. Soc., 159 (2012), p. A1730.

[9] J. N. Reimers and J. R. Dahn, J. of the Electrochem. Soc., 139 (1992), p. 2091.

[10] H. Gabrisch, R. Yazami and B. Fultz, Electrochem. and Solid-State Lett., 5 (2002), p. A111.

[11] M. Qu et al., Adv. Energy Mater., 2 (2012), p. 940.

[12] B. P. Gorman et al., Microscopy Today, 16 (2008), p. 42.

[13] D. R. Diercks et al., J. Electrochem. Soc., 161 (2014), p. F3039.

[14] This work was principally funded by Seed Grants from the Renewable Energy Materials Research Science and Engineering Center, which received its funding from NSF, DMR-0820518. The atom probe used in this research was supported by NSF Award Number 1040456.
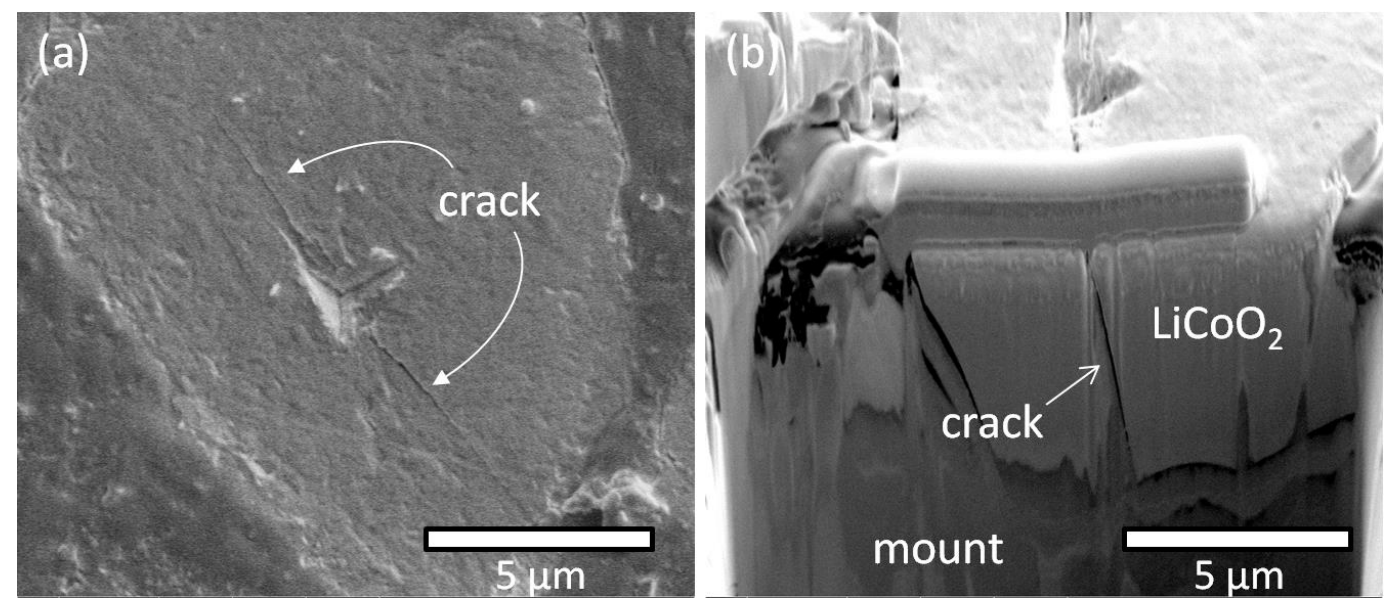

Figure 1. (a) $\mathrm{LiCoO}_{2}$ particle showing a crack across the indented region. (b) Cross-section of the particle from (a) showing that the crack propagated through the entire depth of the particle.
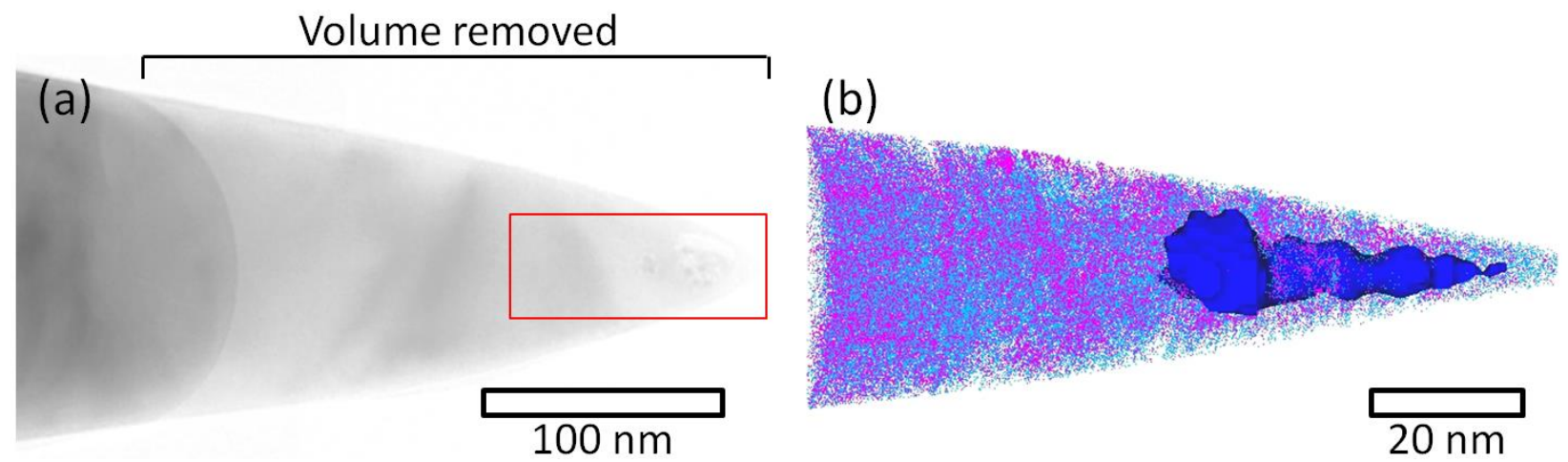

Figure 2. (a) Overlay of TEM images before and after APT analysis indicating the volume of material removed. (b) APT reconstruction of the region indicated by the box in (a). The dark blue surface shows a region enriched in $\mathrm{Co}$ (deficient in $\mathrm{Li}$ ). 